\title{
Pregnancy during the pandemic:
}

\section{The impact of COVID-19-related stress on risk for prenatal depression}

\author{
Lucy S. King ${ }^{1}$, Daisy E. Feddoes ${ }^{1}$, Jacklyn S. Kirshenbaum¹, \\ Kathryn L. Humphreys ${ }^{2}$, \& Ian H. Gotlib ${ }^{1}$ \\ ${ }^{1}$ Department of Psychology, Stanford University \\ 2Deparment of Psychology and Human Development, Vanderbilt University
}

\section{Conflict of Interest}

The authors have no conflicts of interest to report.

\section{Data and Code Availability}

Data and code are available at https://github.com/lucysking/cope_git

\section{Acknowledgements}

Funding was provided by the National Institutes of Health (IHG, R21 MH111978; R21 HD090493); the National Science Foundation (JSK, Graduate Student Research Fellowship); and the Jacobs Foundation (KLH, Early Career Research Fellowship 2017-1261-05). We thank Drs. Moriah Thomason and Michelle VanTiegham at New York University and Drs. Alice Graham and Elinor Sullivan at Oregon Health and Science University for leading the development of the survey materials used in this project. We also thank the participants for their contributions.

\section{Corresponding author:}

Lucy S. King

Department of Psychology, Stanford University

Jordan Hall, 450 Serra Mall, Building 420

Stanford, CA 94305, USA

lucyking@stanford.edu 


\begin{abstract}
Pregnant women may be particularly sensitive to negative events (i.e., adversity) related to the COVID-19 pandemic and affective responses to these events (i.e., stress). We examined COVID-19-related stress and adversity in a sample of 725 pregnant women residing in the San Francisco Bay Area in March-May 2020, 343 of whom provided addresses in California that were geocoded and matched by census tract to measures of community-level risk factors. Women who were pregnant during the pandemic had substantially elevated depressive symptoms compared to matched women who were pregnant prior to the pandemic. Several individual- and community-level risk and protective factors were associated with women's scores on two latent factors of COVID-19-related stress and adversity. Highlighting the role of subjective responses to the pandemic in vulnerability to prenatal depression and factors that influence susceptibility to COVID-19-related stress, these findings inform understanding of the psychosocial sequelae of disease outbreaks among pregnant women.
\end{abstract}




\section{Introduction}

Women experience dramatic metabolic, hormonal, and physical changes during pregnancy and childbirth that occur in synchrony with the rapid development of the fetus (Cárdenas, Kujawa, \& Humphreys, 2020). Because the prenatal period is marked by striking neurobiological plasticity, it is a time of enhanced sensitivity to the environment for both mothers and their fetuses (Bock, Wainstock, Braun, \& Segal, 2015). The novel coronavirus disease 2019 (COVID-19) pandemic is a public health disaster involving widespread increases in exposure to environmental adversity, defined as negative life events related to the environment (e.g., financial losses), as well as psychosocial stress, defined as affective responses to these events (e.g., changes in subjective distress and underlying physiology; Barrero-Castillero, Morton, Nelson, \& Smith, 2019). There is documented inequality in the impact of the COVID-19 pandemic, such that those with historically marginalized identities (e.g., women, people of color, lower income people) are experiencing more numerous and severe social, economic, and psychological challenges due to the pandemic (Fancourt, Steptoe, \& Bu, 2020; O'Connor et al., 2020). Pregnant women are a special population for whom stress and adversity related to the pandemic may have specific characteristics. In particular, pandemic-related stress and adversity may lead to or exacerbate common prenatal mental health difficulties, including depressive symptoms, which, in turn, have been found to negatively influence maternal-child health (Glover, 2014).

Disasters and disease outbreaks are complex humanitarian events that give rise to many forms of individual-level adversity (Beaglehole et al., 2018; Chew, Wei, Vasoo, \& Sim, 2020). Similar to other disasters and epidemics, the COVID-19 pandemic has the potential to increase stress and adversity; however, this pandemic is unprecedented in its characteristics and the reach of its impact (O'Connor et al., 2020). It involves distinct adverse experiences, such as prolonged social isolation due to social distancing requirements, and specific stress responses, such as ongoing fears of contracting a highly virulent virus that, as of late December 
2020, has taken the lives of 1.75 million people. Further, COVID-19 has led to a severe economic recession that disproportionally affects women (Heggeness, 2020; U.S. Bureau of Labor Statistics, 2020). During this time, pregnant women may experience fear for the health of their future infant, worries about their own wellbeing during what is often the most medically vulnerable period of their life, and disruptions to their obstetric care (Preis, Mahaffey, Heiselman, \& Lobel, 2020). These fears are not ungrounded. Pregnant women who contract COVID-19 are more likely to experience severe illness, to die from the virus, to have preterm births, and to experience childbirth-related posttraumatic stress disorder symptoms (Mayopoulos et al., 2020; Woodworth et al., 2020; Zambrano et al., 2020). By taxing the healthcare system, COVID-19 may also indirectly affect pregnant women's health by negatively influencing the prenatal medical care they receive (Roberton et al., 2020). Due to the dual impact of prenatal stress and adversity on women and their fetuses (Van den Bergh et al., 2017), characterizing COVID-19-related stress and adversity in pregnant women is a public health priority. Specifically, by characterizing the nature of pregnant women's experiences of stress and adversity during this pandemic and the impact of these experiences on their psychological health, we can inform policies that help to alleviate women's suffering and prevent its negative consequences on the next generation of children during COVID-19 and future disease outbreaks.

Stress and adversity in any form can be depressogenic (Hammen, 2005), including during or after disasters (Gruebner et al., 2016; Norris, Tracy, \& Galea, 2009; Self-Brown, Lai, Harbin, \& Kelley, 2014). Although research examining the psychological effects of disease outbreaks prior to COVID-19 is limited, studies have documented elevated depressive symptoms in general population samples following the severe acute respiratory syndrome (SARS) and Ebola epidemics (Chew et al., 2020). With respect to COVID-19, emerging findings indicate that depressive symptoms were elevated in the general population compared to historical norms in the weeks following the first shelter-in-place orders in the U.S. and the U.K. 
(Fancourt et al., 2020; Nelson, Pettitt, Flannery, \& Allen, 2020). In a U.S. nationally representative sample, Ettman et al. (2020) found the presence of depressive symptoms during COVID-19 to be $27.8 \%$, compared to $8.5 \%$ in a representative sample assessed prior to the pandemic. Initial studies indicate that $24-33 \%$ of postpartum women have reported clinically significant depression during the pandemic (Cameron et al., 2020; Thayer \& Gildner, 2020; as indicated by scores on the Edinburgh Postnatal Depression Scale [EPDS; Cox, Holden, \& Sagovsky, 1987] $\geq 13$ and $\geq 15$, respectively). From a cumulative risk perspective, pregnant women may be especially vulnerable to experiencing depressive symptoms during COVID-19 because pregnancy itself can be stressful, involving mental and physical preparation for dramatic life changes that can be challenging even when they are welcome (Saxbe, Goldenberg, \& Rossin-Slater, 2018). Thus, for pregnant women, it is possible that COVID-19 is an additional psychosocial burden superimposed on an already challenged system. In this context, pregnant women, as a group, may be more likely to experience elevated depressive symptoms in response to the pandemic than are non-pregnant people, and among pregnant women, those who experience more severe pandemic-related stress and adversity may be at highest risk.

Based on a bioecological model of human development, both proximal processes that vary at the level of the individual and distal factors that vary across community systems influence the nature of individuals' exposure and responses to mass adversity (Bronfenbrenner, 1994; Hoffman \& Kruczek, 2011). For example, whereas some individuals may already have resources in place (e.g., social support) that protect them from stress and adversity related to the COVID-19 pandemic, others may lack these resources and/or have characteristics that predispose them to greater stress and adversity during this time (e.g., physical health conditions; McElroy et al., 2020). Characteristics of one's community, such as the population's health and socioeconomic standing relative to other places, may also affect levels of stress and adversity by influencing social distancing, disease transmission, and mortality (Adhikari et al., 
2020; Jay et al., 2020). Identifying risk and protective factors for stress and adversity related to the COVID-19 pandemic in pregnant women will advance our understanding of who in that population is most vulnerable to the psychosocial sequelae of this pandemic. As the immediate and longer-term consequences of the pandemic continue to unfold, these data will be useful for guiding prenatal care practices based on patient-level variables and for the allocation of public health resources for pregnant women based on the characteristics of communities.

We had four goals in the current study, in which we examined stress and adversity related to the COVID-19 pandemic in a large sample of pregnant women residing predominantly in the San Francisco Bay Area in March-May 2020. In Aim 1, we tested the hypothesis that, compared to women who were pregnant prior to the onset of the COVID-19 pandemic, women who were pregnant during the pandemic have higher self-reported symptoms of depression, indicating that the pandemic has depressogenic effects on pregnant women. In Aim 2, to characterize stress and adversity in pregnant women during the pandemic, we explored the latent structure of pregnant women's reports of stress and adversity related to COVID-19 using dimensionality reduction techniques. In Aim 3, we used a machine-learning approach to identify risk and protective factors that are associated with dimensions of COVID-19 stress and adversity, including both individual-level characteristics (e.g., self-reported social support) and community-level factors (e.g., community health at the census-tract level). Finally, in Aim 4, we examined the associations between dimensions of COVID-19 stress and adversity and women's depressive symptoms, and, using machine-learning, identified the single indicators of stress and adversity that are most strongly associated with prenatal depressive symptoms. Given that the global incidence of infectious disease outbreaks is rising (Smith et al., 2014), the current study elucidates the psychosocial impact on pregnant women of the COVID-19 pandemic specifically, but also informs our understanding of the possible effects of future outbreaks on pregnant women.

\section{Method}




\section{Participants}

Participants included independent samples of pregnant women residing predominantly in the San Francisco Bay Area who participated in the Brain and Behavior Infants Experiences project (BABIES; Humphreys, King, Choi, \& Gotlib, 2018; King, Querdasi, Humphreys, \& Gotlib, 2020) prior to the COVID-19 pandemic (hereafter, the "pre-pandemic" cohort), or who participated in the Stanford COVID-19 Perinatal Experiences (COPE) project following the onset of the COVID-19 pandemic in the United States and state-wide shelter-in-place orders in California (hereafter, the "COVID-19" cohort; see https://osf.io/uqhcv/ for information about the broader COPE project). These projects were separate in their original design and aims.

Pre-pandemic Cohort. Ninety women participated during their pregnancies in a longitudinal study of the association between perinatal experiences and infant and toddler psychobiological development from February 2017-May 2019. Of these women, two did not complete an assessment of their prenatal depressive symptoms and, therefore, were not included in the current analyses. Thus, the final pre-pandemic sample for the current analyses included 88 women. All women resided in the San Francisco Bay Area at the time of study participation.

COVID-19 Cohort. Pregnant women completed an online survey about COVID-19 stress and adversity and current mental health difficulties in April 2020. Of the 1,994 women who responded to the online survey for the Stanford COPE Project, 1,595 were eligible for the broader study based on being currently pregnant or the mother of an infant $<6$ months old. Of eligible women, 1,058 were currently pregnant. 333 of these pregnant women did not complete the entire survey, yielding a final sample for the current analyses of 725 pregnant women. Of the 428 women who provided any information about their current address, 353 resided in California. Of these 353 women, 343 provided their complete current addresses, of whom 333 resided in counties in the Bay Area.

\section{Procedure}


Both the BABIES and COPE projects were approved by the Stanford Institutional Review Board. Participants in both cohorts were recruited during their pregnancies through online advertisements. Advertisements were active for the pre-pandemic cohort in February 2017-May 2019 and during a single week in early April 2020 for the COVID-19 cohort. Data for both cohorts were collected and managed using REDCap electronic data capture tools hosted at Stanford University (Harris et al., 2019, 2009).

Pre-pandemic Cohort. Participants were screened for inclusion/exclusion criteria through a phone interview. Inclusion criteria were that they were currently pregnant, were $\geq$ age 18 years, were fluent in English, and had no immediate plans to leave the geographic area. Exclusion criteria included bipolar disorder, psychosis, or severe learning disabilities that would impair the ability to participate in the study procedures. Further, due to the goals of the broader BABIES project, participants were recruited to be enriched for a history of major depressive disorder (MDD), current MDD, and/or early life adversity. Risk for MDD and early life adversity was evaluated during the phone screening interview using questions from the Center for Epidemiologic Studies Depression scale (Lewinsohn, Seeley, Roberts, \& Allen, 1997) and the Childhood Trauma Questionnaire (Bernstein et al., 1994). Participants who met inclusion/exclusion criteria and agreed to participate attended an in-person session (1-2 hours) in the Department of Psychology at Stanford University where they provided informed written consent and completed a series of questionnaires, interviews, and computer tasks. Participants were compensated for their time.

COVID-19 Cohort. Participants were screened for inclusion/exclusion criteria using questions containing branching logic at the beginning of the online survey. Inclusion criteria for the broader study were that participants were either currently pregnant or had an infant $<6$ months old and were $\geq$ age 18 years. In contrast to the pre-pandemic cohort, participants were unselected for risk for depression or early life adversity. The survey and advertisements were in 
English and also translated into Spanish. Participants who met criteria and agreed to participate provided informed written consent and responded to a series of questions about their experiences related to the pandemic as well as questionnaires measuring symptoms of psychopathology ( 20 minutes). Participants were entered into a random raffle for one of 25 online gift cards.

\section{Measures}

Demographic Characteristics. Participants in both cohorts self-reported their race and ethnicity, level of education, marital status, their country of birth (from which we identified whether they were a U.S. immigrant), their type of housing, their employment status, their annual household income in the previous year, and the number of adults and children in their household. We calculated their income-to-federal poverty line (FPL) ratio based on the number of adults and children in their household. Notably, cost of living in California, especially in the Bay Area, is extremely high; although California is among the wealthier states when considering raw income relative to the FPL, it has the highest rate of poverty in the country when accounting for cost of living (Fox, 2020). Thus, we anticipated that most participants would have household incomes above the FPL. Participants also reported whether or not this was their first pregnancy and their due dates. We calculated gestational weeks based on due date.

Prenatal Depressive Symptoms. Participants in both the pre-pandemic and COVID-19 cohorts completed the EPDS (Cox, Holden, \& Sagovsky, 1987). The EPDS is a self-report questionnaire consisting of 10 items assessing symptoms of depression (e.g., "I have felt sad or miserable") measured on a 4-point scale, yielding total scores ranging from 0 to 30 where higher scores indicate more severe symptoms. Whereas participants in the pre-pandemic cohort completed a modified version of the EPDS in which they were asked to report on their symptoms "during pregnancy," participants in the COVID-19 cohort were asked to report on their symptoms in the "past 7 days." According to a recent meta-analysis, the EPDS has high sensitivity and specificity for detecting depression in pregnant women at a cut-off score of $\geq 11$ 
(Levis, Negeri, Sun, Benedetti, \& Thombs, 2020). Internal consistency of the EPDS quantified with Cronbach's $\alpha$ was good in both cohorts (pre-pandemic cohort=.89, COVID-19 cohort=.85).

COVID-19 Stress and Adversity. Participants in the COVID-19 cohort responded to a series of questions probing their experiences related to the pandemic, developed by Drs. Moriah Thomason and Michelle VanTiegham at New York University and Drs. Alice Graham and Elinor Sullivan at Oregon Health and Science University (see: https://osf.io/uqhcv/). We identified items that asked about adversity (i.e., negative life event related due to the pandemic; e.g., a checklist of changes to prenatal care due to COVID-19, including change from planned vaginal to induction or $\mathrm{C}$-section, cancellation or reduction in prenatal care visits) or psychosocial stress (i.e., affective responses to the pandemic; e.g., "How concerned are you about possible changes to your medical care during your baby's birth as a result of COVID-19"). We did not include items that assessed adversity or stress unrelated to COVID-19 or items that overlapped with symptoms of depression. Overall, we examined 16 indicators of COVID-19 stress and adversity, listed in Table 1 (see Supplementary Material for distributions).

Individual-level risk and protective factors. Using the survey developed by Thomason et al. (https://osf.io/ughcv/), the COVID-19 cohort reported on individual characteristics that may increase risk for or protect against stress and adversity related to the pandemic. These survey items are listed in Table 2 and included, along with several demographic indicators, whether participants had a history of serious medical conditions (e.g., diabetes, lung disease), a history of a mood or anxiety disorder, were at high risk for COVID-19 due to existing medical conditions, their number of existing prenatal medical conditions (e.g., gestational diabetes, hypertension), their number of personal environmental resources (e.g., phone, internet, privacy at home, outdoor space), the extent to which they felt supported by their prenatal care team (not very well supported, somewhat well supported, or very well supported), how well they currently felt supported by their social network (from 1 (Not supported) to 7 (Very supported) and how well they felt supported prior to COVID-19, from which we calculated change in social support 
due to COVID-19 (see Supplementary Material for distributions of each individual-level risk and protective factor).

Community-level risk factors. For participants in the COVID-19 cohort, we first geocoded their current addresses using the Census Geocoding API and the "tidygeocoder" package in $\mathrm{R}$ (Cambon, 2020). We next used the "tigris" package in R (Walker, 2020) to identify the census tract code corresponding to each participant's geolocation. The 343 participants who provided complete addresses in California were then matched based on their census tract codes with population characteristics data from the CalEnviroScreen 3.0 (https://oehha.ca.gov/calenviroscreen/report/calenviroscreen-30), where 333 resided in the Bay Area. We focused on the following 8 community-level indicators of sensitive health and socioeconomic burden: asthma (rate of emergency room (ER) visits for asthma per 10,000 people); cardiovascular disease (rate of ER visits for heart attacks per 10,000 people); low birth weight (\% births of low weight infants in population); low educational attainment (\% of population > age 25 years with < high school education); housing burden (\% of population that is both low income and paying $>50 \%$ of income for housing); linguistic isolation (\% limited English-speaking households); poverty (\% of population with income < twice the federal poverty line); and unemployment (\% of population > age 16 years, eligible for workforce, and unemployed). The indicators were quantified by the census tract percentile relative to the distribution across all 8,057 tracts in California (see Supplementary Material for distributions of each community-level indicator within this sample).

\section{Statistical Analysis}

Statistical analyses were conducted in R version 4.0.2 (R Core Team, 2020) and Mplus version 8 (Muthén \& Muthén, 2017). Data and code for analyses is available at:

\section{https://github.com/lucysking/cope git.}

Aim 1: Examine Prenatal Depressive Symptoms in Matched Samples of Women Pregnant Prior to and During the COVID-19 Pandemic. Analyses for Aim 1 focused on 
subsets of matched participants from the pre-pandemic and COVID-19 cohorts. All matched participants were currently residing in the San Francisco Bay Area at the time of data collection We used Optimal Nearest Neighbor Matching (Stuart, 2010) implemented in the "Matchlt" package in R (Ho, Imai, King, \& Stuart, 2011) to identify matched samples from each cohort with the smallest average absolute distance across all matched pairs. Distance was quantified by the propensity score estimated from a logistic regression of cohort on age, parity, gestational weeks, number of children, marital status, race/ethnicity, income, education, employment status, and history of treatment for mental health difficulties or substance use (see the Supplementary Material for detail methods and results of matching procedures). We used a Welch's $t$-test and multiple ordinary least squares (OLS) regression to test the hypothesis that participants who are pregnant during the COVID-19 pandemic have higher prenatal depressive symptoms than do matched participants who were pregnant prior to the pandemic.

\section{Aim 2: Explore the Latent Structure of COVID-19 Stress and Adversity among}

Pregnant Women. Analyses for Aim 2 focused on the full sample participants in the COVID-19 cohort. We used exploratory factor analysis (EFA) to examine the dimensionality of stress and adversity related to COVID-19. Given that the 16 indicators of stress and adversity (see Table 1) included a mix of continuous normal variables, continuous variables with floor effects (i.e., censored from below), and count variables (modeled with Poisson regression), the EFA was estimated with maximum likelihood robust standard errors using a numerical integration algorithm (MLR; Muthén \& Muthén, 2017). Missing data were handled using Full Information Maximum Likelihood and factors were allowed to correlate using an oblique rotation ("geomin"; Muthén \& Muthén, 2017). Traditional fit statistics for EFA are not calculated when using MLR. Therefore, we determined the number of factors based on chi-squares tests comparing model fit for a model with one additional factor, the Bayesian Information Criterion (BIC) values, and the interpretability of the solution. We removed items with high cross-loadings ( $\geq .30)$ before identifying a final model. We extracted estimated factors scores based on the final EFA solution. 


\section{Aim 3: Identify Individual- and Community-Level Risk and Protective Factors for}

COVID-19 Stress and Adversity in Pregnant Women. Analyses for Aim 3 focused on

participants who resided in California and provided valid addresses that could be geocoded and matched by census tract to the CalEnviroScreen dataset. Among these participants, we used elastic net $(E N)$ regularized regression with the "glmnet" package in R (Jerome, Hastie, Simon, \& Tibshirani, 2010), employing a full EN penalty $(\alpha=.50)$ and an expected gaussian distribution, to explore the associations between potential individual- and community-level variables that may enhance risk or be protective and estimated factor scores for COVID-19 stress and adversity. Specifically, the variables listed in Table 1 were entered as predictors in separate models in which estimated scores for each factor identified in the EFA in Aim 2 served as dependent variables. Given that EN models implement list-wise deletion, participants who were missing values for at least one of the individual-level variables were removed from the analysis, yielding a final sample for Aim 3 of 319 women. We performed leave-one-out cross-validation (LOOCV; James, Hastie, \& Tibshirani, 2013) using the cv.glmnet function to determine the largest $\lambda$ (i.e., hyperparameter, regularization value) associated with the smallest mean-squared error (i.e., "lambda.min"), which yields a sparse matrix of non-zero coefficients. In order to determine model performance, we computed $R^{2}$ based on predicted and observed values. To aid interpretation of the coefficient estimates, we computed zero-order effect sizes with $95 \%$ confidence intervals for the variables that were selected by the EN models.

\section{Aim 4: Examine the Associations of Dimensions of COVID-19 Stress and Adversity} with Prenatal Depressive Symptoms during the COVID-19 Pandemic. We used a multiple OLS linear regression to examine the associations between dimensions of COVID-19 stress and adversity and prenatal depressive symptoms. Specifically, in a single model, prenatal depressive symptoms were regressed on the estimated scores for each factor identified in the EFA in Aim 2. Finally, to identify single variables that may be used in a clinical setting to identify women experiencing the specific types of COVID-19 stress and adversity that are most strongly 
associated with increased risk for prenatal depression, we conducted an additional EN regularized regression to examine the associations between each of the indicators of COVID-19 stress and adversity entered in the EFA model in Aim 2 and prenatal depressive symptoms.

\section{Results}

\section{Sample Characteristics}

In Table 1, we present descriptive statistics for the items related to COVID-19 stress and adversity examined in Aim 2. In Table 2, we present descriptive statistics for the individual- and community-level risk and protective factors examined in Aim 3.

Pre-pandemic cohort: Participants in the pre-pandemic cohort were ages 20-44 years (mean $[S D]=32.55$ [5.04]) and 12-37 gestational weeks (mean gestational age [SD]=24.44 [5.47] weeks). Eighty-three percent were married or partnered and $27 \%$ were primiparous. Participants reported living with 0-4 children (mean $[S D]=0.88$ [1.10]) 1-7 adults household (mean $[S D]=2.38$ [1.12]). They reported their race and ethnicity as follows: 54\% White, $21 \%$ Asian or Asian American, 10\% Hispanic or Latinx, 3\% Black or African American, 3\% Native Hawaiian/Pacific Islander, 1\% Native American/Alaskan Native, and 6\% "other." Although income-to-FPL ratios ranged from $0.06-11.28$, participants tended to have higher incomes relative to national norms ( $8 \%$ had incomes $\leq 200 \%$ of the FPL; mean [SD] income-to-FPL ratio=5.61 [2.52]) and most were highly educated (78\% had $\geq$ a 4 -year college degree). Sixty-eight percent had paid jobs, $17 \%$ were stay-at-home caregivers, $2 \%$ were students, and $10 \%$ were unemployed. Sixty-seven percent of participants had previously received treatment for mental health difficulties or substance abuse. With respect to depressive symptoms, scores on the EPDS ranged from 0-21 (mean $[S D]=7.22$ [5.35]; $25 \%$ of participants scored $\geq$ the cut-off score of 11 , indicating possible depression ( $18 \%$ scored $\geq 13 ; 10 \%$ scored $\geq 15$ ).

COVID-19 cohort: Participants in the COVID-19 cohort were ages 19-50 years (mean $[S D]=33.69$ [4.38]) and between 4-41 gestational weeks (mean [SD]=26.79 [8.81]). Ninetyseven percent were married or partnered and $49 \%$ were primiparous. Participants reported 
living with $0-9$ children (mean $[S D]=0.63[0.93]$ ) and 1-8 adults (mean $[S D]=2.30$ [0.81]). They reported their race and ethnicity as follows: $62 \%$ White, 17\% Asian or Asian American, 11\% Hispanic or Latinx, 7\% multi-racial/multi-ethnic, 1\% Black or African American, $<1 \%$ Native American/Alaskan Native, and $<1 \%$ Native Hawaiian/Pacific Islander. Income-to-FPL ratios ranged from $0.21-14.60$ ( $7 \%$ had incomes $\leq 200 \%$ of the $\mathrm{FPL}$; mean $[S D]$ income-to-FPL ratio = 8.47 [4.13]) and $84 \%$ of participants had $\geq$ a 4 -year college degree. Seventy-six percent had paid jobs, $10 \%$ were stay-at-home caregivers, $2 \%$ were students, $3 \%$ reported "other" types of employment, and $8 \%$ said they were unemployed and looking for work. Forty-one percent of participants had previously received treatment for mental health difficulties or substance abuse. Scores for prenatal depressive symptoms ranged from 0-26 in the full COVID-19 cohort (mean $[S D]=9.54[5.21]) ; 42 \%$ of participants scored $\geq 11$, indicating possible depression ( $29 \%$ scored $\geq$ $13 ; 17 \%$ scored $\geq 15)$. Three participants reported that they had tested positive for COVID-19.

Aim 1: Examine Prenatal Depressive Symptoms in Matched Samples of Women Pregnant Prior to and During the COVID-19 Pandemic

Optimal Nearest Neighbor Matching yielded a subset of 164 women from the prepandemic and COVID-19 cohorts ( $n=82$ from each cohort) who were similar in age, parity, gestational weeks, number of children, marital status, race/ethnicity, income, education, employment status, and history of treatment for mental health difficulties or substance use. The standardized mean difference [SMD] for the propensity scores based on these variables was 0.15 after matching and the variance ratio was 1.66 , indicating acceptable balance between groups (Rubin, 2006; see Supplementary Material for detailed methods and results for matching procedures). Following matching, the groups from each cohort did not differ significantly on any of the variables used in the matching procedure. Because the sample of pre-pandemic participants was recruited to be enriched for a history of MDD or early life adversity, the 82 matched participants from the COVID-19 cohort had higher depressive symptoms (mean $[S D]=10.84[5.64]$ ) than did other participants in this cohort (mean [SD]=9.37 [5.13]; 
$t(99.06)=2.25, p=.027, \operatorname{SMD}[95 \% \mathrm{Cl}]=0.28[0.05,0.51])$, but did not differ significantly in estimated factor scores for COVID-19 objective adversity or subjective stress (see Aim 2). Thus, differences between matched "pre-pandemic" and "COVID-19" participants should be interpreted as differences between two groups of women at relatively higher risk for current depression who differ based on whether they were pregnant prior to or following the onset of the pandemic.

As hypothesized, women who were pregnant during the pandemic had significantly higher levels of prenatal depression than did "pre-pandemic" pregnant women (Welch's $t(161.01)=4.16, p<.001$, Cohen's $d[95 \% \mathrm{Cl}]=.65,[0.33,0.96] ;$ Figure 1). This association held and was similar in effect size when adjusting for age, gestational weeks, marital status, parity, number of children, race/ethnicity, low income status, education, employment status, and history of treatment for mental health difficulties or substance use $(\mathrm{B}=3.17, \mathrm{SE}=0.80, t(152)=3.95, p<$ $.001, \beta[95 \% \mathrm{Cl}]=0.56,[0.28,0.84])$. In addition, participants in the COVID-19 cohort were significantly more likely than were "pre-pandemic" participants to score above the clinical cutoff $(\geq 11)$ on the EPDS indicating possible depression $\left(\chi^{2}(1)=10.31, p=.001\right)$. Whereas $25 \%$ of participants in the pre-pandemic cohort had possible depression, half (51\%) of participants in the COVID-19 cohort had possible depression. Expressed in terms of relative risk, participants in the COVID-19 cohort were twice as likely to have possible depression than were participants in the pre-pandemic cohort. 
Figure 1. Levels of depressive symptoms are significantly higher in pregnant women during the COVID-19 pandemic than in matched pregnant women in a pre-pandemic cohort. $\mathrm{N}=166$ (83 in each group). This figure shows, for each prenatal cohort, levels of prenatal depressive symptoms for each participant (dots), the medians and interquartile ranges of prenatal depressive symptoms (box plots), and the distribution of prenatal depressive symptoms (flat violin plots).

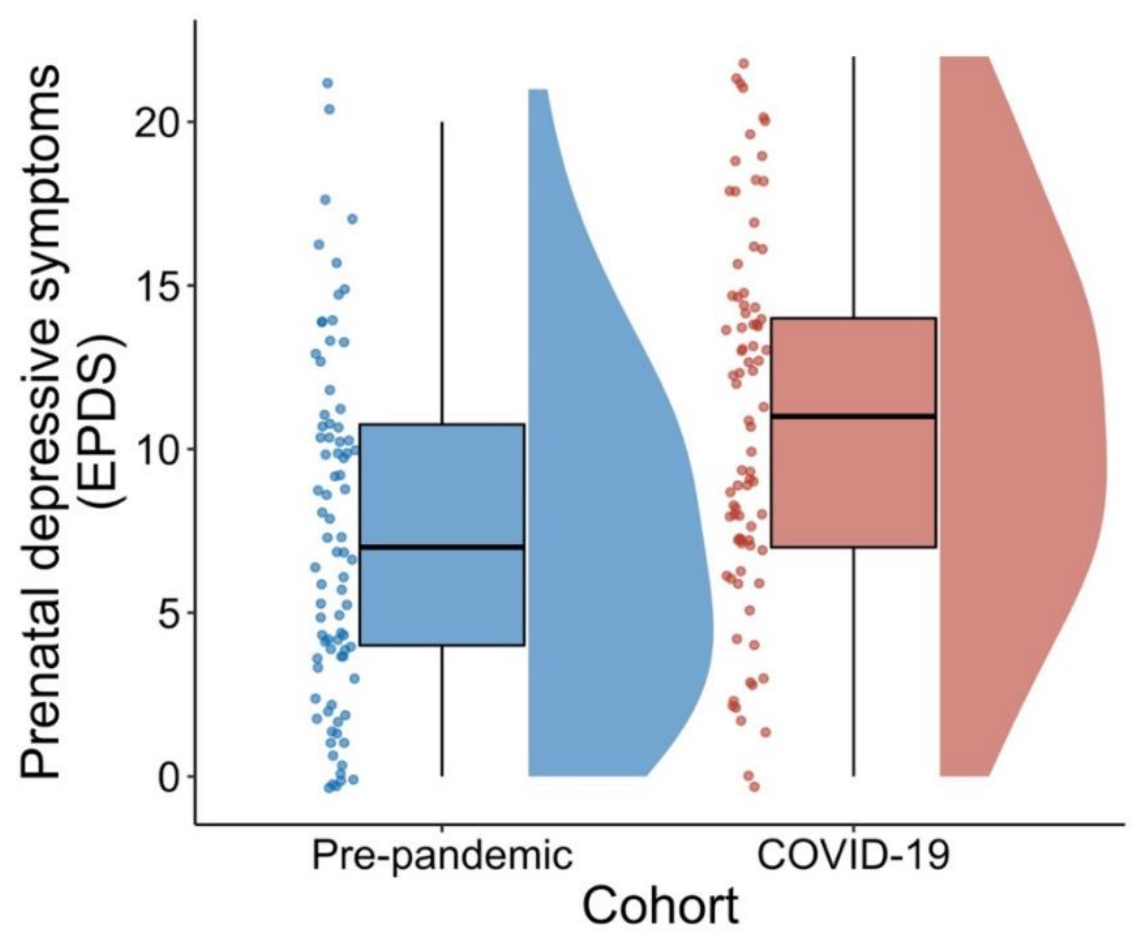

Aim 2: Explore the Latent Structure of COVID-19 Stress and Adversity among Pregnant Women

We compared models with one, two, and three factors (models with $>3$ factors did not converge) for the 16 of items related to stress and adversity listed in Table 1. Based on chisquare tests of model fit and the BIC values, more complex models provided better fits to the data. Specifically, a 2 -factor solution $(\mathrm{BIC}=43,752.83)$ fit better than a 1 -factor solution $\left(B I C=44,252.81 .16 ; \chi^{2}(15)=581.11, p<.001\right)$, and a 3-factor solution $(B I C=43,425.15)$ fit better than a 2 -factor solution $\left(\chi^{2}(14)=461.49, p<.001\right)$. When items with high oblique rotated crossloadings (>|.30|) were removed (distress due to current financial/employment impacts, distress due to expected financial/employment impacts, and restrictions on activity), leaving 13 items in 
the model, we observed a similar pattern. Specifically, the 2-factor solution provided a significantly better fit to the data than the 1 -factor solution $\left(\chi^{2}(12)=591.18, p<.001\right)$, and the BIC was reduced in the 2-factor compared to the 1-factor model and in the 3-factor model compared

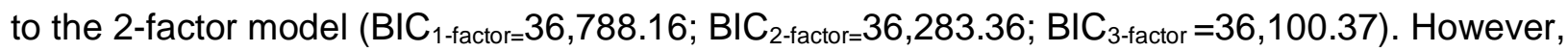
the 3-factor model remained uninterpretable due high cross-loadings. In contrast, the 2-factor solution contained no high cross-loadings and was interpretable.

In order to maximize both model fit and interpretability, we selected the 2 -factor solution from the model of the 13 indicators of COVID-19 stress and adversity presented with their factor loadings in Figure 2. The two factors were correlated at $r=.41$. The first factor, which we labeled objective COVID-19 adversity, was defined by three items assessing extant or expected negative life events due to COVID-19, including the number of current changes in the participant's or the participant's partner's employment and finances (e.g., loss of job, loss of health insurance, reduced ability to afford childcare), the number of expected changes in employment and finances, and the number of current changes to the participant's prenatal care (e.g., changed from planned vaginal birth to induction or $\mathrm{C}$-section, change in prenatal care providers, cancellation or reduction of prenatal care visits).

The second factor, which we labeled subjective COVID-19 stress, was defined by 10 items assessing affective responses to COVID-19. Items that loaded onto this factor included those assessing the severity of participants' concerns about the impact of COVID-19 on the social support and medical care they would receive during labor and delivery, their infant's health, and their capacity to care for their infant following birth. Items assessing the severity of participants' distress due to COVID-19 also loaded on this factor, including distress due to the possibility that they or their family would contract COVID-19, the potential for reduced access to material resources (e.g., baby supplies), health care, and positive social interactions because of COVID-19, and distress due to current disruptions in social interactions because of COVID-19. 
Finally, participants' ratings of the overall impact of COVID-19 on their daily life and the valence of this impact loaded onto this subjective stress factor.

We extracted the estimated factor scores based on the solution presented in Figure 2 to be used in analyses for Aims 2-3. Scores for each factor were normally distributed (see Supplementary Material). 
Table 1. Descriptive statistics for indicators of COVID-19 stress and adversity. For valence of overall impact, lower scores indicate that the impact of the pandemic was viewed as having a more negative impact on life.

\begin{tabular}{lcc}
\hline \multicolumn{1}{c}{ Variable } & Mean (SD) & Range \\
\hline Changes to prenatal care & $2.29(1.39)$ & $0-6$ \\
Concern for birth medical care & $4.06(2.40)$ & $0-7$ \\
Concern for birth social support & $4.96(2.14)$ & $0-7$ \\
Concern for caregiving capacity & $3.52(2.85)$ & $0-7$ \\
Concern for infant's health & $4.04(2.66)$ & $0-7$ \\
Self-quarantine/government restrictions & $1.44(0.62)$ & $0-4$ \\
Current employment/financial impacts & $4.71(2.88)$ & $0-19$ \\
Expected employment/financial impacts & $3.87(3.69)$ & $0-22$ \\
Distress due to current financial impacts & $2.69(2.06)$ & $0-6$ \\
Distress due to expected financial impacts & $2.98(2.08)$ & $0-6$ \\
Distress due to potential family illness & $3.31(2.02)$ & $0-6$ \\
Distress due to own potential illness & $1.86(1.99)$ & $0-6$ \\
Distress due to reduced access & $1.47(0.69)$ & $0-3$ \\
Distress due to social disruptions & $2.76(1.94)$ & $0-6$ \\
Overall impact to daily life & $4.14(1.21)$ & $0-6$ \\
Valence of overall impact & $-1.74(1.04)$ & $-3-3$ \\
\hline
\end{tabular}


Figure 2. Factor loadings from exploratory factor analysis of pregnant women's reports of stress and adversity related to COVID-19.

\begin{tabular}{|c|c|c|}
\hline & $\begin{array}{c}\text { Factor 1: } \\
\text { Objective Adversity }\end{array}$ & $\begin{array}{c}\text { Factor 2: } \\
\text { Subjective Stress }\end{array}$ \\
\hline Current employment/financial impacts & 1.02 & -0.06 \\
\hline Changes to prenatal care & 1.00 & -0.01 \\
\hline Expected employment/financial impacts & 0.99 & 0.02 \\
\hline Distress due to family illness & -0.08 & 0.64 \\
\hline Distress due to own illness & -0.13 & 0.62 \\
\hline Distress due to reduced access & 0.07 & 0.56 \\
\hline Concern for infant's health & -0.03 & 0.55 \\
\hline Concern for birth medical care & -0.01 & 0.54 \\
\hline Concern for birth social support & 0.01 & 0.51 \\
\hline Overall impact to daily life & 0.14 & 0.48 \\
\hline Distress due to social disurptions & -0.01 & 0.47 \\
\hline Concern for caregiving capacity & 0.07 & 0.46 \\
\hline Valence of overall impact & -0.11 & -0.46 \\
\hline
\end{tabular}




\section{Aim 3: Identify Individual- and Community-Level Risk and Protective Factors for COVID- 19 Stress and Adversity in Pregnant Women}

First, we entered the 25 individual- and community-level variables listed in Table 2 in an EN regression model in which estimated factor scores of objective COVID-19 adversity were the dependent variable. Next, we repeated this analysis with estimated factor scores of subjective COVID-18 stress as the dependent variable. Results are presented in Table 2 and Figures 4 and 5 .

The LOOCV of the EN model of objective COVID-19 adversity identified an optimal $\lambda=.04$, resulting in 11 variables with non-zero coefficients that explained $11 \%$ of the variance in objective adversity. Based on zero-order effect size, women who were immigrants to the U.S, had histories of a mood/anxiety disorders, and had more prenatal medical complications were higher in objective adversity. Although the associations were weaker in effect size, women who received greater support from their prenatal care team and had more environmental resources were lower in objective adversity.

The LOOCV of the EN model of subjective COVID-19 stress identified an optimal $\lambda=.07$, resulting in 12 variables with non-zero coefficients that explained $18 \%$ of the variance in subjective stress. Based on zero-order effect size, women who were at high risk for COVID-19 due existing medical conditions, were persons of color, were primiparous, had histories of a mood/anxiety disorder, resided in census tracts that were higher relative to other tracts in the percent of the population with less than a high school education, and resided in census tracts where a greater percent of the population was limited English-speaking were higher in subjective stress. In contrast, women with more environmental resources, who received greater support from their prenatal care team, and who experienced less deterioration or greater improvement in social support from prior to during the pandemic were lower in subjective stress. 
Table 2. Descriptive statistics, elastic net estimates, and zero-order effect sizes for individual- and community-level risk and protective factors. Descriptive statistics are for the 343 participants who provided valid addresses in California. Elastic net and effect size estimates are for the 319 participants included in the elastic net regression. FPL=Federal Poverty Line. Objective adversity and subjective stress are estimated factor scores from solution presented in Figure 2. Zero-order effect sizes are Cohen's $d$ values for discrete variables, Pearson's $r$ values continuous variables., and Spearman's $\rho$ values for ordinal or count data.

\begin{tabular}{|c|c|c|c|c|}
\hline \multirow{2}{*}{ Variable } & \multirow{2}{*}{$\begin{array}{l}\mathrm{N}(\%) \text { or } \\
\text { Mean (SD) }\end{array}$} & \multirow{2}{*}{ Range } & \multicolumn{2}{|c|}{$\begin{array}{c}\text { Elastic Net Estimate / } \\
\text { zero-order } \\
\text { effect size }[95 \% \text { Cl] }\end{array}$} \\
\hline & & & $\begin{array}{c}\text { Objective } \\
\text { Adversity } \\
R^{2=} .11\end{array}$ & $\begin{array}{c}\text { Subjective } \\
\text { Stress } \\
R^{2=} .18\end{array}$ \\
\hline \multicolumn{5}{|c|}{ Individual-level Factors } \\
\hline Primiparous & $173(50)$ & & $\begin{array}{c}0.10 \\
.15[-.07, .37]\end{array}$ & $\begin{array}{c}0.13 \\
.28[.06, .50]\end{array}$ \\
\hline $\begin{array}{l}\text { Previous miscarriage } \\
\text { or abortion }\end{array}$ & $106(31)$ & & & \\
\hline $\begin{array}{l}\text { History of serious } \\
\text { medical conditions }\end{array}$ & $80(23)$ & & & \\
\hline $\begin{array}{l}\text { History of mood or anxiety } \\
\text { disorder }\end{array}$ & $93(27)$ & & $\begin{array}{c}0.04 \\
.20[-.05, .45]\end{array}$ & $\begin{array}{l}0.03 \\
.26[.01, .51]\end{array}$ \\
\hline $\begin{array}{l}\text { High risk for COVID-19 due to } \\
\text { existing medical conditions }\end{array}$ & $83(24)$ & & & $\begin{array}{c}0.10 \\
.44[.18, .70]\end{array}$ \\
\hline Person of color & $136(39)$ & & & $.31[.09, .54]$ \\
\hline Immigrant to U.S. & $69(20)$ & & $\begin{array}{c}0.07 \\
.20[-.07, .47]\end{array}$ & \\
\hline \multicolumn{5}{|l|}{ Low income according to FPL } \\
\hline Multi-bedroom housing & $279(81)$ & & & \\
\hline Age & & & $\begin{array}{c}0.04 \\
.06[-.05, .17]\end{array}$ & $\begin{array}{c}0.03 \\
.03[-.08, .14]\end{array}$ \\
\hline Environmental resources & $4.21(0.85)$ & $0-5$ & $\begin{array}{c}-0.11 \\
-.11[-.21,<.01]\end{array}$ & $\begin{array}{c}-0.10 \\
-.16[-.26,-.05]\end{array}$ \\
\hline Prenatal medical conditions & $0.22(0.48)$ & $0-2$ & & \\
\hline Support from prenatal care team & $1.60(0.56)$ & $0-2$ & $\begin{array}{c}-0.12 \\
-.14[-.24,-.03]\end{array}$ & $\begin{array}{c}-0.10 \\
-.13[-.23,-.02]\end{array}$ \\
\hline Current social support & $3.91(1.58)$ & $0-6$ & & \\
\hline Change in social support & $-0.64(1.46)$ & $-5-4$ & & $\begin{array}{c}-0.11 \\
-.16[-.27,-.05]\end{array}$ \\
\hline \multicolumn{5}{|c|}{ Community-level Factors } \\
\hline Rate of ER visits for asthma & $39.13(28.65)$ & $0.02-99.61$ & $\begin{array}{c}0.05 \\
.07[-.04, .18]\end{array}$ & \\
\hline $\begin{array}{l}\text { Rate of ER visits for } \\
\text { heart attacks }\end{array}$ & $29.52(23.85)$ & $0.32-95.72$ & & \\
\hline Rate of low weight births & $43.73(27.64)$ & $0.00-99.82$ & $\begin{array}{c}0.01 \\
.05[-.06, .16]\end{array}$ & \\
\hline$\%$ low educational attainment & $32.40(24.22)$ & $0.18-91.72$ & & $\begin{array}{c}0.13 \\
.15[.05, .25]\end{array}$ \\
\hline$\%$ housing burdened & $39.87(25.58)$ & $0.27-98.95$ & & \\
\hline \% limited English-peaking & $47.94(26.27)$ & $0.00-99.34$ & $\begin{array}{c}0.07 \\
.06[-.05, .17]\end{array}$ & $\begin{array}{l}0.01 \\
.11[.01, .29]\end{array}$ \\
\hline$\%$ in poverty & $30.79(21.67)$ & $0.24-90.80$ & $\begin{array}{l}-0.09 \\
-.04[-.15, .07]\end{array}$ & $\begin{array}{l}-0.03 \\
.03[-.08, .14]\end{array}$ \\
\hline$\%$ unemployed & $29.53(22.60)$ & $0.04-98.45$ & & \\
\hline
\end{tabular}


Figure 4. Zero-order associations between risk and protective factors selected in elastic net regression of estimated factor scores for objective COVID-19 adversity. $d$ = Cohen's standardized mean difference. $\rho=$ Spearman's rank-order correlation. $r=$ Pearson's correlation. Values within brackets are lower and upper bounds of $95 \%$ confidence intervals.

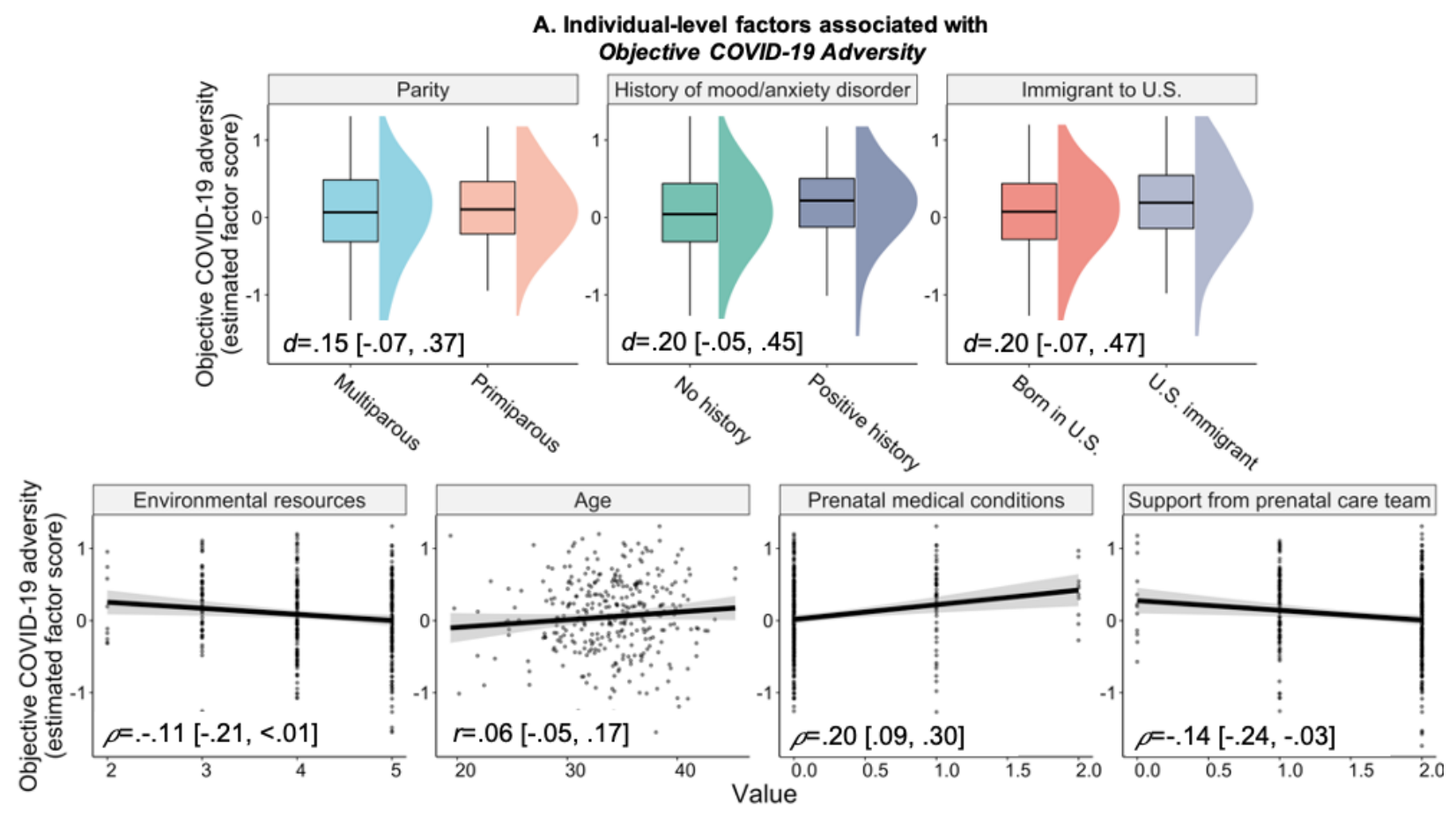

B. Community-level factors associated with Objective COVID-19 Adversity
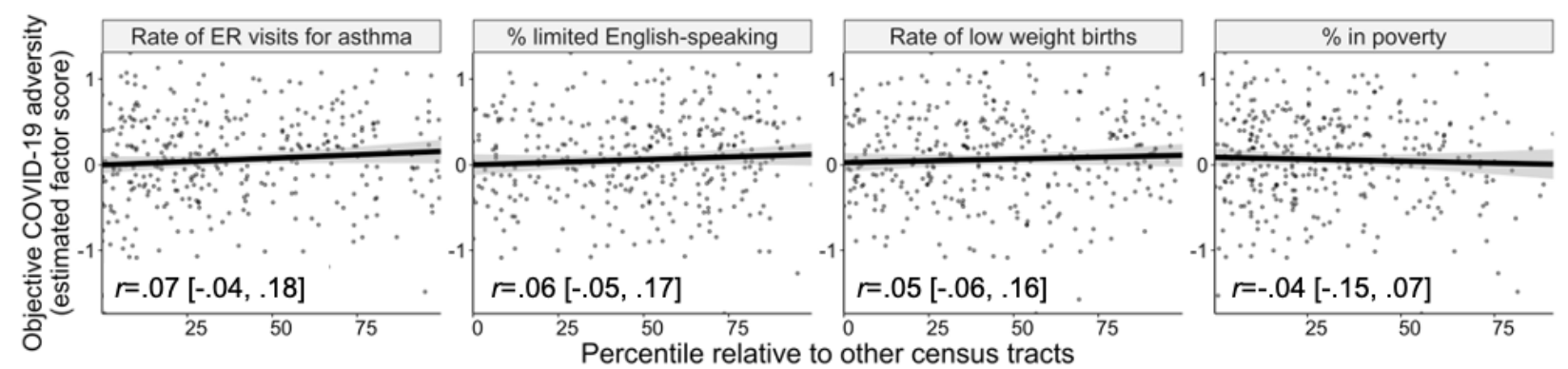
Figure 5. Zero-order associations between risk and protective factors selected in elastic net regression of estimated factor scores for subjective COVID-19 stress. $d=$ Cohen's standardized mean difference. $\rho=$ Spearman's rank-order correlation. $r=$ Pearson's correlation. Values within brackets are lower and upper bounds of $95 \%$ confidence intervals.

A. Individual-level factors associated with Subjective COVID-19 Stress

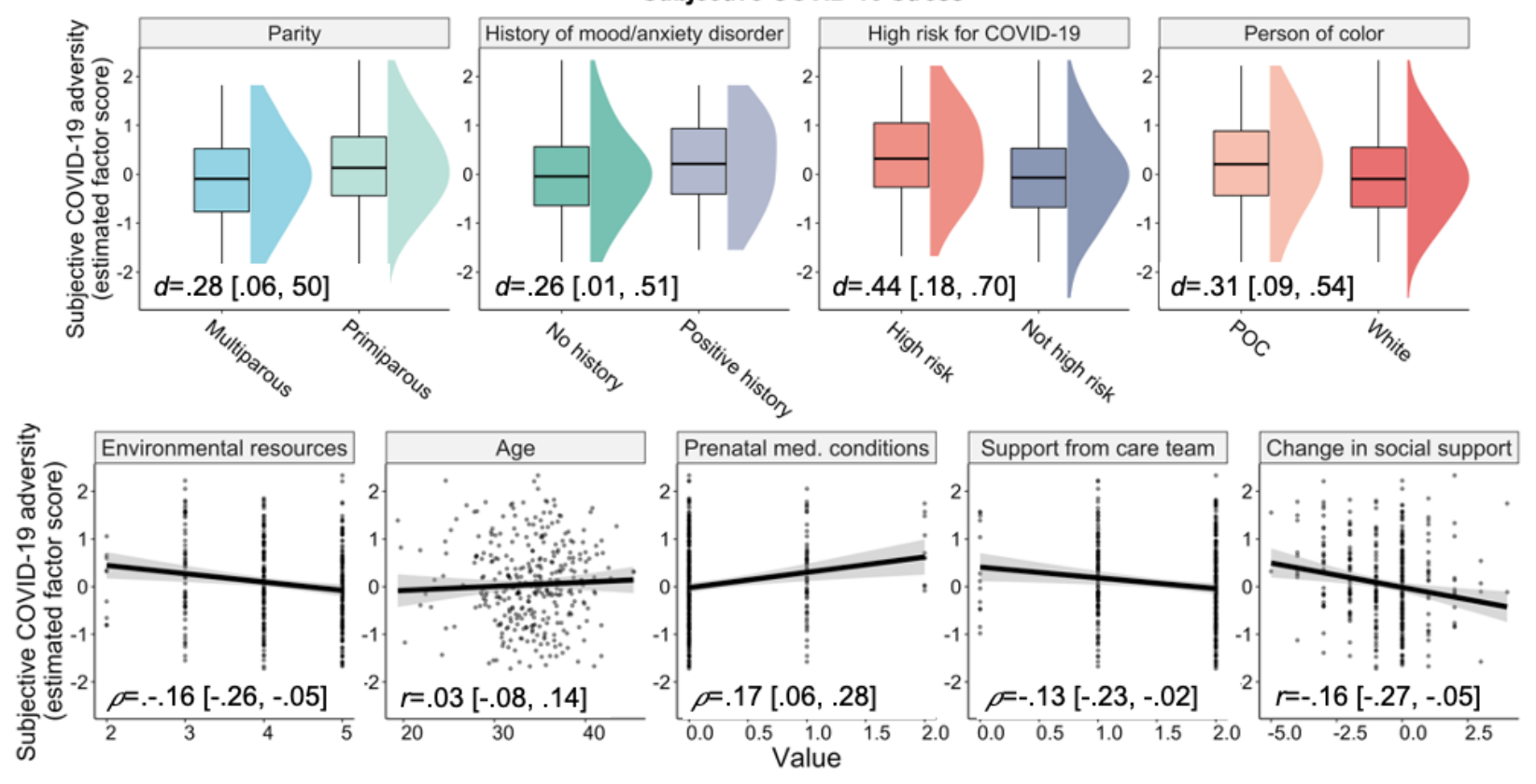

B. Community-level factors associated with Subjective COVID-19 Adversity

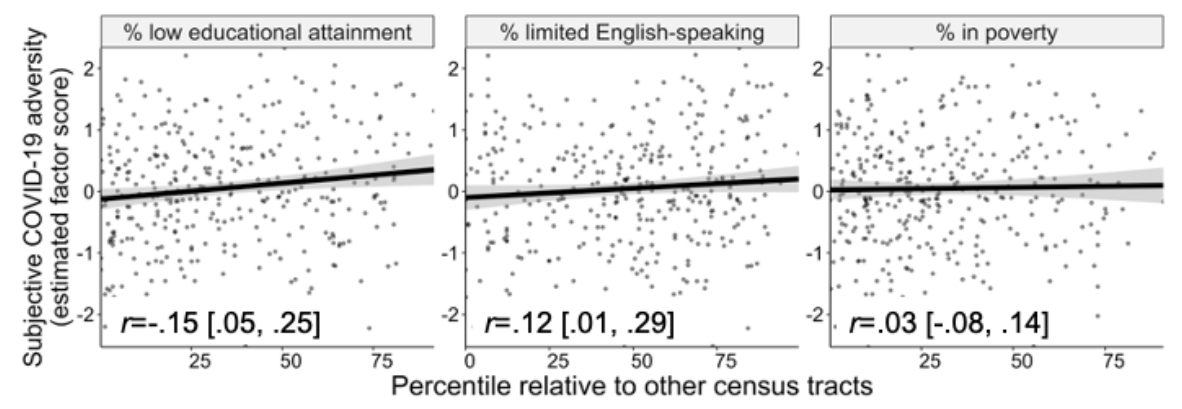




\section{Aim 4: Examine the Associations of Dimensions of COVID-19 Stress and Adversity with Prenatal Depressive Symptoms during the COVID-19 Pandemic}

In the full sample of participants in the COVID-19 cohort and when covarying for scores of objective COVID-19 adversity, women who were higher in subjective COVID-19 stress had significantly higher prenatal depressive symptoms $(\beta=0.44,95 \% \mathrm{Cl}[0.35,0.52], p<.001$, $\left.R^{2}=.17\right)$. In contrast, although the zero-order association between objective COVID-19 adversity and prenatal depressive symptoms was statistically significant $(r=0.24, p<.001)$, women's scores on this factor were not significantly associated with their symptoms when covarying for their subjective stress $(\beta=0.04,95 \% \mathrm{Cl}[-0.04,0.12], p=.356)$.

Finally, we sought to identify single indicators of COVID-19 stress and adversity that were most strongly associated with prenatal depressive symptoms. Given that factor scores are difficult to interpret, identifying single indicators may aid in characterizing our findings and translating them to clinical settings. The LOOCV for the EN model of depressive symptoms identified an optimal $\lambda=.02$, resulting in 9 variables with non-zero coefficients, 7 of which were variables that had loaded onto the subjective COVID-19 stress factor and two of which were variables that had loaded onto the objective COVID-19 adversity factor. We present the associations between each of these variables and prenatal depressive symptoms in Figure 7. Listed in order of zero-order effect size, these variables were as follows: distress due to social disruptions (EN estimate $=0.20)$, distress due to reduced access to resources (EN estimate $=0.18$ ), severity of overall impact of COVID-19 on daily life (EN estimate $=-0.06)$, valance of overall impact of COVID-19 (EN estimate=-0.11), concern about the availability of social support during birth (EN estimate $=0.16)$, distress that family would contract virus (EN estimate $=0.04$ ), distress that participant would contract virus (EN estimate $=0.02)$, current employment/financial impacts (EN estimate $=0.09$ ), number of changes to prenatal care due to COVID-19 (EN estimate=0.02). 
Figure 6. Zero-order associations between estimated factor scores for COVID-19 stress and adversity and prenatal depressive symptoms. When estimated scores for both factors were entered together, only Subjective COVID-19 stress remained significantly associated with prenatal depressive symptoms.

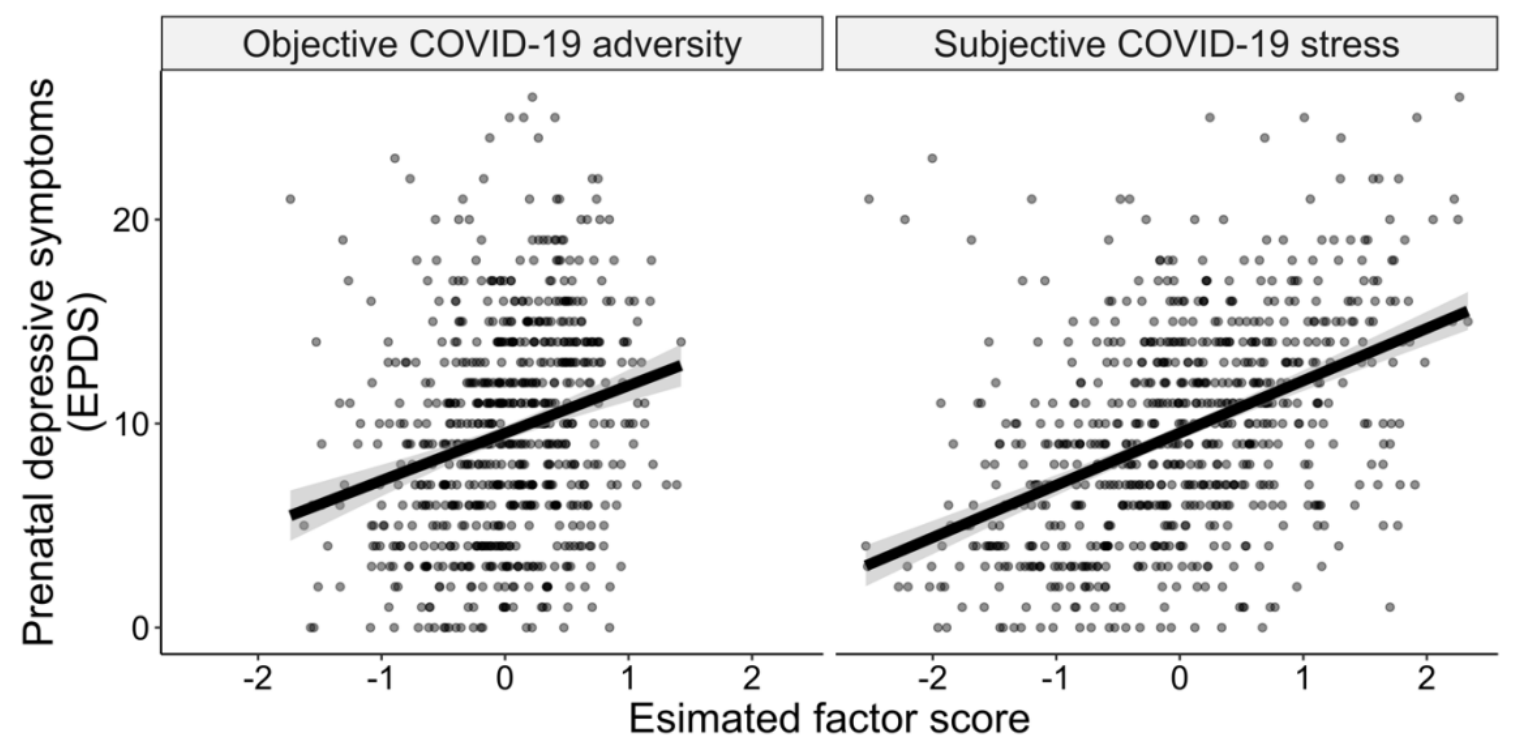


Figure 7. Zero-order between single indicators of COVID-19 stress and adversity selected in elastic net regression of prenatal depressive symptoms. $\rho=$ Spearman's rank-order correlation. $r=$ Pearson's correlation. Values within brackets are lower and upper bounds of $95 \%$ confidence intervals

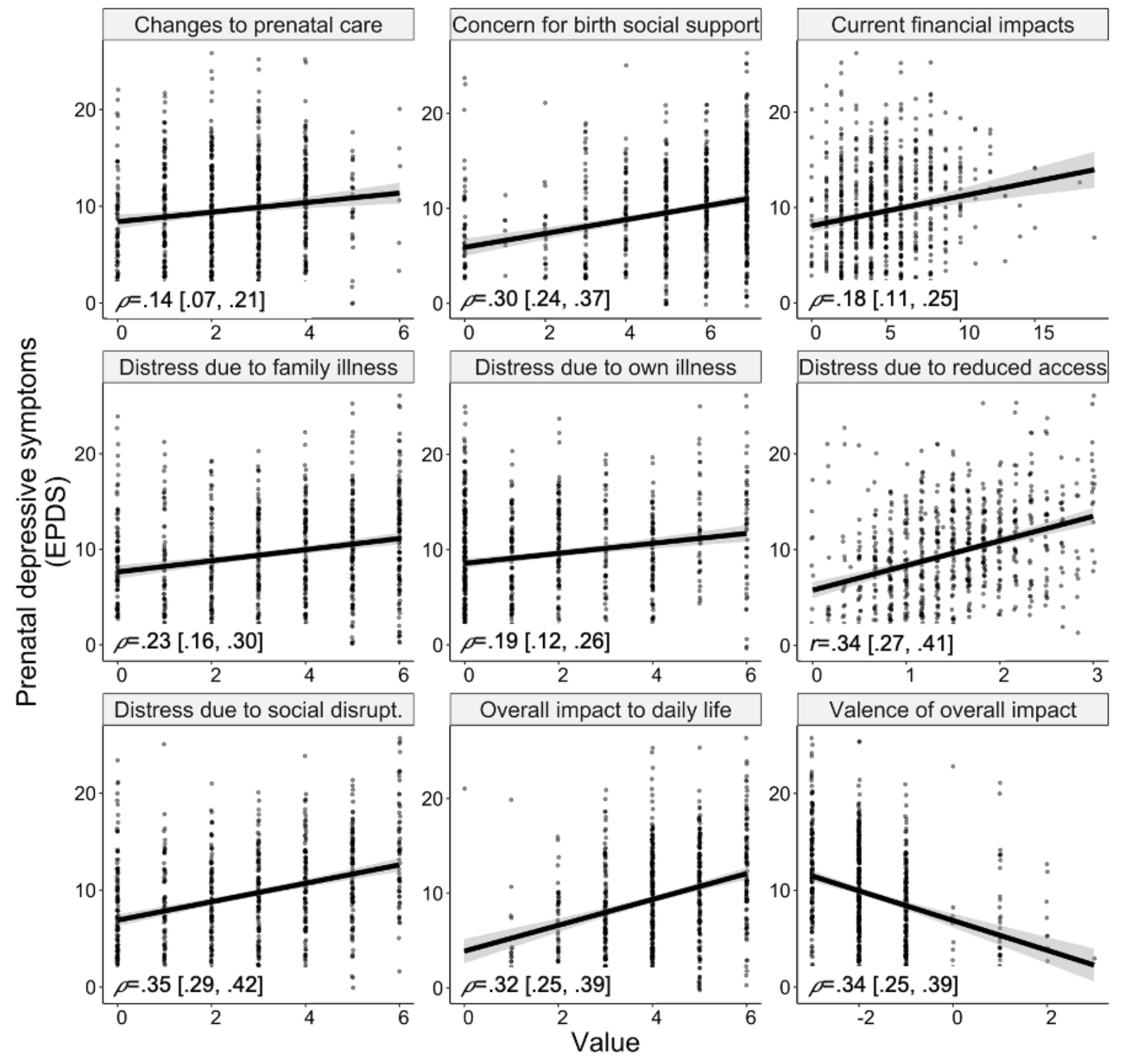




\section{Discussion}

We examined pregnant women's experiences of the COVID-19 pandemic following the initial "shelter-in-place" response in the San Francisco Bay Area. Distinguishing between adversity (i.e., negative life events) and stress (i.e., affective responses to negative life events; Barrero-Castillero et al., 2019), we explored the latent structure of women's experiences of the pandemic and searched for individual- and community-level factors that may increase risk or protect against stress and adversity related to COVID-19. We investigated whether women who were pregnant during this period had elevated depressive symptoms compared to a matched sample of women who were pregnant prior to the pandemic. Finally, we examined whether women who experienced more severe stress and adversity related to the pandemic had more severe depressive symptoms and identified the specific forms of stress and adversity that were most strongly associated with their symptoms.

Similar to previous studies of the impact of the COVID-19 pandemic in the general population (Ettman et al., 2020; Nelson et al., 2020), we found evidence that the onset of the pandemic led to increased depressive symptoms specifically among pregnant women. Concerningly, compared to historical levels of prenatal depression, the discrepancy in depressive symptoms during COVID-19 that we observed in pregnant women is even greater than has been found when comparing general population samples during COVID-19 to prepandemic samples. In the full sample of 725 pregnant women that we surveyed in March-May 2020 , over $40 \%$ reported symptoms indicating possible depression when using a cutoff score of $\geq 11$ on the EPDS (Levis et al., 2020). In comparison, a systematic review of studies including over 35,000 women conducted in developed countries prior to the pandemic found that the average rate of prenatal depression was $17 \%$ (Underwood, Waldie, D'Souza, Peterson, \& Morton, 2016). Moreover, supporting our hypotheses, we found that compared to a matched sample of women who were pregnant prior to the pandemic, women who were pregnant during the pandemic were twice as likely to have possible depression. 
Although, outside the context of COVID-19, major depression is no more common in pregnant women than in non-pregnant women (Ashley, Harper, Arms-Chavez, \& LoBello, 2016), pregnant women may be especially vulnerable to the depressogenic effects of stress and adversity related to the pandemic due to the dramatic biological and psychosocial changes that occur during pregnancy (Cárdenas et al., 2020). Given this plasticity, elevations in depressive symptoms in pregnancy, even when temporary, may have lasting effects on maternal and fetal neurobiology. In fact, the transition to parenthood is theorized to be a "critical window for adult health" that may shape trajectories for physical and psychological health in midlife (Saxbe et al., 2018). For example, depression in pregnancy has been associated with changes in maternal prenatal inflammation, indicated by elevations in pro-inflammatory cytokines (Gustafsson et al., 2018; Osborne et al., 2018). In turn, maternal inflammatory biomarkers have been linked with subsequent infant stress responses and negative affect (Gustafsson et al., 2018; Osborne et al., 2018) and variation in neonatal brain structure and function (Rasmussen et al., 2019; Rudolph et al., 2018). Women who experience prenatal depression may also behave differently with their infants. For example, they are more likely to cease exclusive breastfeeding earlier in the postpartum period (Figueiredo, Canário, \& Field, 2014) and to report poorer bonding with their infants postnatally (Rossen et al., 2016). Because elevations in prenatal depressive symptoms may have enduring consequences for maternal-child health, policymakers and clinicians should consider measures to support women who were pregnant during this pandemic even if their depressive symptoms have since remitted.

A critical step in understanding the relation between the impact of the pandemic and risk for prenatal depression is to characterize the nature of women's experiences during this period. We found that women's experiences related to COVID-19 fell along the two dimensions of objective COVID-19 adversity (i.e., extant or expected negative life events due to the pandemic) and subjective COVID-19 stress (i.e., affective responses to negative life events due to the pandemic). Experiences of objective adversity due to COVID-19 included those that are 
common to the general population (e.g., changes to finances and employment) and those that specifically affect pregnant women (e.g., changes to prenatal care). Similarly, subjective stress due to COVID-19 involved women's fears that they or their family would contract the virus, as well as concerns about the medical care and social support they would receive during labor and delivery. We found that, above and beyond their experiences of objective adversity due to COVID-19, women who experienced more severe subjective stress had significantly higher depressive symptoms. In fact, subjective stress due to COVID-19 explained 17\% of the variance in women's depressive symptoms.

Our findings are consistent with those of other recent studies that have distinguished between the impacts of objective exposure to adversity and subjective stress severity on risk for psychopathology. For example, subjective reports, rather than objective measures, appear to explain risk for psychopathology following childhood maltreatment (Danese \& Widom, 2020). Perceived socioeconomic standing is more strongly associated with subjective wellbeing than is objectively measured income and education-a finding that is not solely explained by common method variance (Tan, Kraus, Carpenter, \& Adler, 2020). Most relevant to the current study, Nelson et al. (2020) found in a general population sample from across the U.S., Canada, and Europe, that individuals' levels of subjective concern about COVID-19 were more strongly associated with their depressive symptoms than were epidemiological data signifying objective risk (e.g., country confirmed case). Nonetheless, objective changes to the environment, including widespread disruptions in the functioning of society due to COVID-19 and the real threats that this virus poses to maternal-child health (Mayopoulos et al., 2020; Woodworth et al., 2020; Zambrano et al., 2020) create the conditions for susceptible individuals to experience subjective stress. Our findings suggest that in addition to policies that help to mitigate disruptions to the environment due to the pandemic (e.g., measures to inhibit the transmission of the virus, payments to families for recovery of financial losses), treatments that focus on 
cognitions about the self and the environment may help to alleviate depressive symptoms in pregnant women during this period.

With implications for the allocation of resources to support recovery from the psychosocial sequelae of the pandemic, we identified individual- and community-level factors that were associated with risk for the experience of objective adversity and subjective stress during this period. Specifically, women who were immigrants to the U.S., who had a history of a mood or anxiety disorder, and who had prenatal medical conditions (e.g., gestational diabetes, small fetal size) reported greater objective adversity due to COVID-19. Women who had histories of mood or anxiety disorders and who had existing prenatal medical conditions also reported more severe subjective stress. Additional risk factors for more severe subjective stress included identifying as a person of color, having a medical condition that increased one's risk of contracting COVID-19, and living in communities that had higher rates of low education and linguistic isolation. It is well-documented that the pandemic has had an unequal impact in terms of transmission and mortality; members of our society who are medically sensitive or experience systemic discrimination are most at risk for contracting the virus and dying from it (KaracaMandic, Georgiou, \& Sen, 2020). Supporting the results of another recent study (Preis et al., 2020), our findings suggest that, even in the initial months following the onset of the pandemic in the U.S., women who were already experiencing challenges due to socioeconomic inequality and mental and physical health were the most vulnerable to experiencing adversity (i.e., disruptions to their finances, employment, and obstetric care) and stress (i.e., fears and concerns about the impact of the pandemic).

It is important to consider limitations of the current study. This was a convenience sample of women recruited through online advertisements in the San Francisco Bay Area. Therefore, our sample is only representative of women with access to the internet who live in this geographic region. Due to our study design, it is likely that we did not capture the experiences of some individuals who may be the most severely exposed to stress and adversity 
related to COVID-19 (e.g., individuals in severe poverty, individuals who do not have time to devote to participating in research). Although our study was racially and ethnically diverse, few Black or Indigenous women participated. In addition, the sample for our study tended to be highly educated, likely reflecting the demands, due to high cost of living, of residing in the Bay Area. Although we matched women who were surveyed following the onset of the COVID-19 pandemic to women who were pregnant prior to the pandemic in order to draw inferences about the potential impact of the pandemic on depressive symptoms, it is possible that additional unmeasured variables differentiate these two groups of women (e.g., personality characteristics linked to willingness to participate in online versus in-person research). Importantly, we used a cross-sectional observational design in this study. Therefore, we cannot conclude that the onset of the pandemic caused increases in women's depressive symptoms, nor can we speak to how women's experiences of stress, adversity, and depressive symptoms have changed over time as the pandemic has unfolded. In light of recent data from the U.K. indicating that depressive symptoms declined within-individuals across the first 20 weeks following the initial "lockdown" response, it is possible that many of the women who participated in the current study have since recovered from their initially high levels of symptoms. Nonetheless, the U.S. has remained among the highest risk countries for COVID-19 since March 2019; as of late December 2020, California has the highest daily case rate in the nation.

Overall, these findings suggest that pregnant women experienced elevated levels of depressive symptoms following California's "shelter-in-place" measures initiated in response to the COVID-19 pandemic in March 2020. Both individual- and community-level factors tied to social and economic inequality appeared to enhance vulnerability to stress and adversity related to COVID-19. Above and beyond their experiences of financial hardship, disruptions to employment, and changes to their prenatal care, women's subjective stress responses, including the degree to which they felt distressed about the impact of the pandemic on their and their future infants' lives, were associated with their depressive symptoms. Unfortunately, prior 
to COVID-19, the U.S. already lagged behind other countries in its treatment of pregnant women, mothers, and infants. Among similarly developed countries, the U.S. has the highest maternal and child mortality rates (Gunja, Fitzgerald, \& Zephyrin, 2020; Thakrar, Forrest, Maltenfort, \& Forrest, 2018) and is the only developed country without a paid family leave policy (U.S. Department of Labor, 2012). Given the possibility that even temporary increases in depressive symptoms during pregnancy may have long-lasting consequences for maternalchild health, it is critical that research with pregnant women exposed to the psychosocial impact of COVID-19 continues even as policy-makers consider essential and significant support for pregnant and postpartum women during this period and beyond. 


\section{References}

Adhikari, S., Pantaleo, N. P., Feldman, J. M., Ogedegbe, O., Thorpe, L., \& Troxel, A. B. (2020). Assessment of community-level disparities in Coronavirus Disease 2019 (COVID-19) infections and deaths in large US metropolitan areas. JAMA Network Open, 3(7), e2016938. https://doi.org/10.1001/jamanetworkopen.2020.16938

Ashley, J. M., Harper, B. D., Arms-Chavez, C. J., \& LoBello, S. G. (2016). Estimated prevalence of antenatal depression in the US population. Archives of Women's Mental Health, 19(2), 395-400. https://doi.org/10.1007/s00737-015-0593-1

Barrero-Castillero, A., Morton, S. U., Nelson, C. A., \& Smith, V. C. (2019). Psychosocial stress and adversity: Effects from the perinatal period to adulthood. NeoReviews, 20(12), e686e696. https://doi.org/10.1542/neo.20-12-e686

Beaglehole, B., Mulder, R. T., Frampton, C. M., Boden, J. M., Newton-Howes, G., \& Bell, C. J. (2018). Psychological distress and psychiatric disorder after natural disasters: Systematic review and meta-analysis. British Journal of Psychiatry, 213(6), 716-722. https://doi.org/10.1192/bjp.2018.210

Bernstein, D. P., Fink, L., Handelsman, L., Foote, J., Lovejoy, M., Wenzel, K., ... Ruggiero, J. (1994). Initial reliability and validity of a new retrospective measure of child abuse and neglect. American Journal of Psychiatry, 151(8), 1132-1136. https://doi.org/10.1176/ajp.151.8.1132

Bock, J., Wainstock, T., Braun, K., \& Segal, M. (2015). Stress In utero: Prenatal programming of brain plasticity and cognition. Biological Psychiatry, 78(5), 315-326. https://doi.org/10.1016/j.biopsych.2015.02.036

Bronfenbrenner, U. (1994). Ecological models of human development. In International Encyclopedia of Education (2nd ed.). Oxford: Elsevier.

Cambon, J. (2020). tidygeocoder: Geocoding made easy. Retrieved from https://jessecambon.github.io/tidygeocoder/ 
Cameron, E. E., Joyce, K. M., Delaquis, C. P., Reynolds, K., Protudjer, J. L. P., \& Roos, L. E. (2020). Maternal psychological distress \& mental health service use during the COVID-19 pandemic. Journal of Affective Disorders, 276, 765-774. https://doi.org/10.1016/j.jad.2020.07.081

Cárdenas, E. F., Kujawa, A., \& Humphreys, K. L. (2020). Neurobiological changes during the peripartum period: implications for health and behavior. Social Cognitive and Affective Neuroscience, 15(10), 1097-1110. https://doi.org/10.1093/scan/nsz091

Chew, Q. H., Wei, K. C., Vasoo, S., \& Sim, K. (2020). Narrative synthesis of psychological and coping responses towards emerging infectious disease outbreaks in the general population: Practical considerations for the COVID-19 pandemic. The Journal of Clinical Psychiatry, 81(6), 350-356. https://doi.org/10.4088/JCP.20r13450

Cox, J. L., Holden, J. M., \& Sagovsky, R. (1987). Detection of postnatal depression. Development of the 10-item Edinburgh Postnatal Depression Scale. The British Journal of Psychiatry: The Journal of Mental Science, 150, 782-786.

Danese, A., \& Widom, C. S. (2020). Objective and subjective experiences of child maltreatment and their relationships with psychopathology. Nature Human Behaviour, 4(8), 811-818. https://doi.org/10.1038/s41562-020-0880-3

Ettman, C. K., Abdalla, S. M., Cohen, G. H., Sampson, L., Vivier, P. M., \& Galea, S. (2020). Prevalence of depression symptoms in US adults before and during the COVID-19 pandemic. JAMA Network Open, 3(9), e2019686. https://doi.org/10.1001/jamanetworkopen.2020.19686

Fancourt, D., Steptoe, A., \& Bu, F. (2020). Trajectories of anxiety and depressive symptoms during enforced isolation due to COVID-19: longitudinal analyses of 36,520 adults in England. The Lancet Psychiatry, 0366(20), 1-9. https://doi.org/10.1101/2020.06.03.20120923

Figueiredo, B., Canário, C., \& Field, T. (2014). Breastfeeding is negatively affected by prenatal 
depression and reduces postpartum depression. Psychological Medicine, 44(5), 927-936. https://doi.org/10.1017/S0033291713001530

Fox, L. (2020). The Supplemental Poverty Measure: 2019. The US Census Bureau (Vol. 8). Retrieved from https://www.census.gov/library/publications/2020/demo/p60-272.html

Glover, V. (2014). Maternal depression, anxiety and stress during pregnancy and child outcome; What needs to be done. Best Practice and Research: Clinical Obstetrics and Gynaecology, 28(1), 25-35. https://doi.org/10.1016/j.bpobgyn.2013.08.017

Gruebner, O., Lowe, S. R., Tracy, M., Joshi, S., Cerdá, M., Norris, F. H., .. Galea, S. (2016). Mapping concentrations of posttraumatic stress and depression trajectories following Hurricane Ike. Scientific Reports, 6(May), 1-12. https://doi.org/10.1038/srep32242

Gunja, M. Z., Fitzgerald, M., \& Zephyrin, L. (2020). Maternal mortality and maternity care in the United States compared to 10 other developed countries. Retrieved from https://www.commonwealthfund.org/publications/issue-briefs/2020/nov/maternal-mortalitymaternity-care-us-compared-10-countries

Gustafsson, H. C., Sullivan, E. L., Nousen, E. K., Sullivan, C. A., Huang, E., Rincon, M., ... Loftis, J. M. (2018). Maternal prenatal depression predicts infant negative affect via maternal inflammatory cytokine levels. Brain Behavior and Immunity, 73(June), 470-481. https://doi.org/10.1016/j.bbi.2018.06.011

Hammen, C. (2005). Stress and depression. Annual Review of Clinical Psychology, 1(1), 293319. https://doi.org/10.1146/annurev.clinpsy.1.102803.143938

Harris, P. A., Taylor, R., Minor, B. L., Elliot, V., Fernandez, M., O’Neal, L., .. REDCap Consortium. (2019). The REDCap consortium: Building an international community of software partners. Journal of Biomedical Informatics, 95. https://doi.org/https://doi.org/10.1016/j.jbi.2019.103208

Harris, P. A., Taylor, R., Thielke, R., Payne, J., Gonzales., N., \& Conde, J. G. (2009). Research electronic data capture (REDCap) - A metadata-driven methodology and workflow process 
for providing translational research informatics suppor. Journal of Biomedical Informatics, 42(2), 377-381. https://doi.org/https://doi.org/10.1016/j.jbi.2008.08.010

Heggeness, M. L. (2020). Estimating the immediate impact of the COVID-19 shock on parental attachment to the labor market and the double bind of mothers. Review of Economics of the Household, (33). https://doi.org/10.1007/s11150-020-09514-x

Ho, D. E., Imai, K., King, G., \& Stuart, E. A. (2011). Matchlt: Nonparametric preprocessing for parametric causal inference. Journal of Stastical Software, 42(8), 1-28. https://doi.org/10.18637/jss.v042.i08

Hoffman, M. a., \& Kruczek, T. (2011). A bioecological model of mass trauma: Individual, community, and societal effects. The Counseling Psychologist, 39(8), 1087-1127. https://doi.org/10.1177/0011000010397932

Humphreys, K. L., King, L. S., Choi, P., \& Gotlib, I. H. (2018). Maternal depressive symptoms, self-focus, and caregiving behavior. Journal of Affective Disorders, 238, 465-471. https://doi.org/10.1016/j.jad.2017.07.051

James, G., Hastie, T., \& Tibshirani, R. (2013). Resampling methods. In An Introduction to Statistical Learning. Springer Texts in Statistics, vol 103. New York, NY: Springer. https://doi.org/10.1007/978-1-4614-7138-7_5

Jay, J., Bor, J., Nsoesie, E. O., Lipson, S. K., Jones, D. K., Galea, S., \& Raifman, J. (2020). Neighbourhood income and physical distancing during the COVID-19 pandemic in the United States. Nature Human Behaviour. https://doi.org/10.1038/s41562-020-00998-2 Jerome, F., Hastie, T., Simon, N., \& Tibshirani, R. (2010). Regularization paths for generalized linear models via coordinate descent. Journal of Statistical Software, 33(1), 1-22. Retrieved from http://www.jstatsoft.org/v33/i01/

Karaca-Mandic, P., Georgiou, A., \& Sen, S. (2020). Assessment of COVID-19 hospitalizations by race/ethnicity in 12 states. JAMA Internal Medicine, 19-21. https://doi.org/10.1001/jamainternmed.2020.3857 
King, L., Querdasi, F. R., Humphreys, K., \& Gotlib, I. (2020). Dimensions of the language environment in infancy and symptoms of psychopathology in toddlerhood. OSF Preprints, 1-40. https://doi.org/10.31219/OSF.IO/CAQEZ

Levis, B., Negeri, Z., Sun, Y., Benedetti, A., \& Thombs, B. D. (2020). Accuracy of the Edinburgh Postnatal Depression Scale (EPDS) for screening to detect major depression among pregnant and postpartum women: Systematic review and meta-analysis of individual participant data. BMJ, 371, m4022. https://doi.org/10.1136/bmj.m4022

Lewinsohn, P., Seeley, J. R., Roberts, R. E., \& Allen, N. B. (1997). Center for Epidemiologic Studies Depression Scale (CES-D) as a screening instrument for depression among community-residing older adults. Psychology and Aging, 12(2), 277-287.

Mayopoulos, G. A., Ein-dor, T., Dishy, G. A., Nandru, R., Chan, S. J., Hanley, L. E., ... Dekel, S. (2020). COVID-19 is associated with traumatic childbirth and subsequent mother-infant bonding problems. Journal of Affective Disorders. https://doi.org/10.1016/j.jad.2020.12.101

McElroy, E., Patalay, P., Moltrecht, B., Shevlin, M., Shum, A., Creswell, C., \& Waite, P. (2020). Demographic and health factors associated with pandemic anxiety in the context of COVID-19. British Journal of Health Psychology, 25(4), 934-944. https://doi.org/10.1111/bjhp.12470

Muthén, L. K., \& Muthén, B. O. (2017). Mplus User’s Guide. (8th ed.). Los Angeles, CA: Muthén \& Muthén.

Nelson, B. W., Pettitt, A., Flannery, J. E., \& Allen, N. B. (2020). Rapid assessment of psychological and epidemiological correlates of COVID-19 concern, financial strain, and health-related behavior change in a large online sample. PloS One, 15(11), e0241990. https://doi.org/10.1371/journal.pone.0241990

Norris, F. H., Tracy, M., \& Galea, S. (2009). Looking for resilience: Understanding the longitudinal trajectories of responses to stress. Social Science \& Medicine, 68(12), 21902198. https://doi.org/10.1016/j.socscimed.2009.03.043 
O’Connor, D. B., Aggleton, J. P., Chakrabarti, B., Cooper, C. L., Creswell, C., Dunsmuir, S., ... Armitage, C. J. (2020). Research priorities for the COVID-19 pandemic and beyond: A call to action for psychological science. British Journal of Psychology, 111(4), 603-629. https://doi.org/10.1111/bjop.12468

Osborne, S., Biaggi, A., Chua, T. E., Du Preez, A., Hazelgrove, K., Nikkheslat, N., ... Pariante, C. M. (2018). Antenatal depression programs cortisol stress reactivity in offspring through increased maternal inflammation and cortisol in pregnancy: The Psychiatry Research and Motherhood - Depression (PRAM-D) Study. Psychoneuroendocrinology, (December 2017), 1-11. https://doi.org/10.1016/j.psyneuen.2018.06.017

Preis, H., Mahaffey, B., Heiselman, C., \& Lobel, M. (2020). Vulnerability and resilience to pandemic-related stress among U.S. women pregnant at the start of the COVID-19 pandemic. Social Science and Medicine, 266(September), 113348. https://doi.org/10.1016/j.socscimed.2020.113348

R Core Team. (2020). R: A language and environment for statistical computing. Vienna, Austria: R Foundation for Statistical Computing. Retrieved from https://www.r-project.org/ Rasmussen, J. M., Graham, A. M., Entringer, S., Gilmore, J. H., Styner, M., Fair, D. A., ... Buss, C. (2019). Maternal Interleukin-6 concentration during pregnancy is associated with variation in frontolimbic white matter and cognitive development in early life. Neurolmage, (185), 825-835. https://doi.org/10.1016/j.neuroimage.2018.04.020

Roberton, T., Carter, E. D., Chou, V. B., Stegmuller, A. R., Jackson, B. D., Tam, Y., ... Walker, N. (2020). Early estimates of the indirect effects of the COVID-19 pandemic on maternal and child mortality in low-income and middle-income countries: a modelling study. The Lancet Global Health, 8(7), e901-e908. https://doi.org/10.1016/S2214-109X(20)30229-1

Rossen, L., Hutchinson, D., Wilson, J., Burns, L., A Olsson, C., Allsop, S., ... Mattick, R. P. (2016). Predictors of postnatal mother-infant bonding: the role of antenatal bonding, maternal substance use and mental health. Archives of Women's Mental Health, 19(4), 
609-622. https://doi.org/10.1007/s00737-016-0602-z

Rubin, D. B. (2006). Using propensity scores to help design observational studies: Application to the tobacco litigation. Matched Sampling for Causal Effects, 365-382. https://doi.org/10.1017/CBO9780511810725.030

Rudolph, M. D., Graham, A. M., Feczko, E., Miranda-Dominguez, O., Rasmussen, J. M., Nardos, R., ... Fair, D. A. (2018). Maternal IL-6 during pregnancy can be estimated from newborn brain connectivity and predicts future working memory in offspring. Nature Neuroscience, 21(5), 765-772. https://doi.org/10.1038/s41593-018-0128-y

Saxbe, D., Goldenberg, D., \& Rossin-Slater, M. (2018). The transition to parenthood as a critical window for adult health. American Psychologist, 73(9), 0-11.

Self-Brown, S., Lai, B. S., Harbin, S., \& Kelley, M. Lou. (2014). Maternal posttraumatic stress disorder symptom trajectories following Hurricane Katrina: An initial examination of the impact of maternal trajectories on the well-being of disaster-exposed youth. International Journal of Public Health. https://doi.org/10.1007/s00038-014-0596-0

Smith, K. F., Goldberg, M., Rosenthal, S., Carlson, L., Chen, J., Chen, C., \& Ramachandran, S. (2014). Global rise in human infectious disease outbreaks. Journal of the Royal Society Interface, 11(101), 1-6. https://doi.org/10.1098/rsif.2014.0950

Stuart, E. A. (2010). Matching methods for causal inference: A review and a look forward. Statistical Science, 25(1), 1-21. https://doi.org/10.1214/09-STS313

Tan, J. J. X., Kraus, M. W., Carpenter, N. C., \& Adler, N. E. (2020). The association between objective and subjective socioeconomic status and subjective well-being: A meta-analytic review. Psychological Bulletin, 146(11), 970-1020. https://doi.org/10.1037/bul0000258

Thakrar, A., Forrest, A., Maltenfort, M., \& Forrest, C. (2018). Child mortality In the US and 19 OECD comparator nations: A 50-Year time-trend analysis. Health Affairs, 37(1).

Thayer, Z. M., \& Gildner, T. E. (2020). COVID-19-related financial stress associated with higher likelihood of depression among pregnant women living in the United States. American 
Journal of Human Biology, (July), 1-5. https://doi.org/10.1002/ajhb.23508

U.S. Bureau of Labor Statistics. (2020). Employment situation summary -- October 2020. Retrieved from https://www.bls.gov/news.release/empsit.nr0.htm

U.S. Department of Labor. (2012). National Compensation Survey: Employee benefits in the United States, March 2012 (Bulletin No. 2773). Retrieved from www.bls.gov/ncs/ebs/benefits/2012/ebbl0050.pdf

Underwood, L., Waldie, K., D'Souza, S., Peterson, E. R., \& Morton, S. (2016). A review of longitudinal studies on antenatal and postnatal depression. Archives of Women's Mental Health, 19(5), 711-720. https://doi.org/10.1007/s00737-016-0629-1

Van den Bergh, B. R. H., van den Heuvel, M. I., Lahti, M., Braeken, M., de Rooij, S. R., Entringer, S., ... Schwab, M. (2017). Prenatal developmental origins of behavior and mental health: The influence of maternal stress in pregnancy. Neuroscience and Biobehavioral Reviews, S0149-7634(16), 1-39. https://doi.org/10.1016/j.neubiorev.2017.07.003

Walker, K. (2020). tigris: Load census TIGER/line shapefiles. Retrieved from https://cran.rproject.org/package=tigris

Woodworth, K. R., Olsen, E. O., Neelam, V., Lewis, E. L., Galang, R. R., Oduyebo, T., ... Whitehill, F. (2020). Birth and infant outcomes following laboratory-confirmed SARS-CoV-2 infection in pregnancy - SET-NET, 16 Jurisdictions, March 29-October 14, 2020. MMWR. Morbidity and Mortality Weekly Report, 69(44), 1635-1640. https://doi.org/10.15585/mmwr.mm6944e2

Zambrano, L. D., Ellington, S., Strid, P., Galang, R. R., Oduyebo, T., Tong, V. T., ... Zapata, L. (2020). Update: Characteristics of symptomatic women of reproductive age with laboratoryconfirmed SARS-CoV-2 infection by pregnancy status — United States, January 22October 3, 2020. MMWR. Morbidity and Mortality Weekly Report, 69(44), 1641-1647. https://doi.org/10.15585/mmwr.mm6944e3 
